Revue d'histoire de l'Amérique française

DEVVUE D.HISTOIRE DE L'AMÉRIQUE FRANÇAISE

\title{
L'histoire partisane : Maurice Duplessis et son temps vus par Robert Rumilly et Conrad Black
}

\section{René Durocher}

Volume 31, numéro 3, décembre 1977

URI : https://id.erudit.org/iderudit/303636ar

DOI : https://doi.org/10.7202/303636ar

Aller au sommaire du numéro

Éditeur(s)

Institut d'histoire de l'Amérique française

ISSN

0035-2357 (imprimé)

1492-1383 (numérique)

Découvrir la revue

Citer cette note

Durocher, R. (1977). L'histoire partisane : Maurice Duplessis et son temps vus par Robert Rumilly et Conrad Black. Revue d'histoire de l'Amérique française, 31(3), 407-426. https://doi.org/10.7202/303636ar d'utilisation que vous pouvez consulter en ligne. 
NOTE CRITIQUE

\section{L'HISTOIRE PARTISANE: MAURICE DUPLESSIS ET SON TEMPS \\ VUS \\ PAR ROBERT RUMILLY ET CONRAD BLACK*}

RENÉ DUROCHER

Département d'bistoire

Université de Montréal

Depuis quelques années on assiste à un renouveau d'intérêt chez les Québécois pour leur histoire, en particulier pour celle qui touche à la période contemporaine. On le voit, entre autres, par l'intérêt que suscite le personnage de Maurice Duplessis. Depuis quelque temps il a eu droit à une pièce de théâtre, une série de quatorze émissions à la radio, une autre série de sept heures à la télévision, au dévoilement d'une statue par nul autre que René Lévesque et à deux grandes biographies de plus de mille pages chacune par Robert Rumilly et Conrad Black.

Ces deux biographies monumentales publiées récemment et largement diffusées dans le public méritent de retenir notre attention à cause de leur ampleur et de la nature de la documentation dont les deux auteurs ont disposé. Rumilly et Black sont les deux seuls chercheurs ayant eu accès à l'ensemble du fonds Duplessis qui est toujours la propriété des amis de l'ex-premier ministre. De plus, ils ont interrogé de nombreux témoins de l'époque.

Duplessis a été et reste, dix-huit ans après sa mort, un personnage fascinant et très controversé dans l'histoire du Québec. On pouvait espérer qu'avec le recul du temps et une solide documen- 
tation, les deux auteurs nous aideraient à mieux connaître et apprécier l'œuvre de cet homme important. Même si nous apprenons beaucoup de choses en lisant ces ouvrages, l'extrême esprit partisan des auteurs rend ces œuvres très décevantes.

Robert Rumilly, depuis fort longtemps, s'est identifié comme un partisan de l'Union Nationale et un admirateur de Maurice Duplessis. Les derniers tomes de son Histoire de la Province de Québec de même que certains de ses pamphlets et son ouvrage de propagande intitulé Quinze années de réalisations, publié en 1956, ne laissent aucun doute à ce sujet.

Conrad Black est un avocat et un homme d'affaires multimillionnaire. Il possède une chaîne d'une vingtaine de journaux et il siège au conseil d'administration de grandes corporations dont Argus, Eaton, la Banque impériale, etc. Son intérêt pour Duplessis lui est venu de ses études en histoire à Carleton et à McGill et sans doute aussi du fait qu'il a coudoyé les milieux de l'Union Nationale alors qu'il rédigeait des discours pour le premier ministre Daniel Johnson. Ajoutons que ce jeune homme n'a que trente-trois ans et que son œuvre sur Duplessis est très influencée par celle de Rumilly pour qui il a beaucoup d'admiration et dont il partage plusieurs points de vue.

Il convenait d'analyser ces deux ouvrages dans une même note car ils ont le même objet et s'appuient essentiellement sur les mêmes sources. De plus, leur œuvre procède de la même conception de l'histoire; leur interprétation générale de Duplessis et de son époque est identique et la filiation entre les deux auteurs est évidente. Cette comparaison des deux ouvrages est très éclairante parce qu'elle permet de comprendre d'une manière saisissante et révélatrice comment chacun des auteurs a exploité la documentation mise à sa disposition.

Les deux auteurs souhaitent nous faire découvrir Maurice Duplessis et son époque. Plus encore, ils veulent réhabiliter la mémoire de l'ancien Premier ministre, victime selon eux, de viles calomnies de son vivant et après sa mort. C'est une motivation qui en vaut d'autres pour s'intéresser à un personnage historique, mais elle est un peu étroite et certainement dangereuse.

Rumilly et Black n'échappent pas au piège qui guette les biographes lorsqu'ils cherchent à expliquer une époque entière par l'étude des faits et gestes d'un homme. Que Duplessis ait joué un rôle important au Québec de 1936 à 1959, tous en conviennent. Mais il faut 
quand même éviter de tout mesurer à l'aune de Duplessis et de tout ramener à sa personne. L'absence de rigueur et le parti pris des auteurs en faveur de leur héros ne font qu'aggraver singulièrement le danger de nous donner une vision déformée de cette période cruciale de l'histoire du Québec. Même si Black est plus critique que Rumilly, tous deux nous présentent Duplessis comme un grand homme sur le plan personnel et sur le plan politique; son régime est le meilleur qu'ait connu le Québec et son époque fut celle de la «vraie» révolution tranquille.

Rumilly suit son personnage de sa naissance à sa mort et son interprétation se retrouve disséminée avec art, au fil de son récit. Duplessis naît dans un milieu qui le prépare parfaitement à son futur rôle de Premier ministre. L'auteur nous présente le père de Maurice comme un homme politique important et de grand talent, mais le récit qu'il nous fait de sa carrière (I: 12 et ss) ne paraît guère justifier ce point de vue. Le jeune Duplessis fait ses études au Collège Notre-Dame où il se lie d'amitié avec le célèbre frère André puis, d'étape en étape, il devient avocat. Il se dégage du récit l'image d'un jeune Duplessis très brillant, non seulement bien préparé mais prédestiné à son futur rôle politique. Vers 1914-1915 alors qu'il exerce sa profession d'avocat à Trois-Rivières, l'auteur nous dit: «Maurice Duplessis continue sa vie réglée» (I: 33 ).

Conrad Black, lui, nous présente un Duplessis bien différent lorsqu'il écrit en parlant du héros: «Les excès et les débauches qui caractérisent la vie qu'il mena depuis son départ du collège jusqu'à ce qu'il renonce aux boissons alcooliques à cinquantre-trois ans...» (I: 27). Plus loin, Black revient sur ce sujet: «Sa présence parmi les solides buveurs qui se rassemblaient aux endroits à la mode pour boire et raconter des histoires salées était devenue habituelle. L'automobile qu'il conduisait, une grosse Winton, était une voiture de luxe achetée à crédit au grand désarroi de son père.» (I: 31.) Certes, Rumilly dans son récit concède que Duplessis quelquefois prenait «un verre de gin ou deux» ou même "un verre de gin de trop». Il ne manque pas d'ajouter cependant que jamais, sauf une fois à Shawinigan en 1937, Duplessis n'est entré à l'Assemblée ou n'a participé à une cérémonie officielle alors qu'il était sous l'influence de l'alcool (I: 577). Pourtant Black écrit que dans la période 1936-1939, le premier ministre Duplessis «consommait une grande quantité de boissons alcooliques et était fréquemment ivre, grossier et agressif lors de cérémonies publiques d'importance secondaire» (I: 359). 
C'est ainsi que la conduite de Duplessis sera expliquée bien différemment par les deux auteurs en certaines occasions. Par exemple, lors du discours d'ouverture de la campagne électorale à TroisRivières, en octobre 1939, Duplessis commet un impair politique grave en se prononçant non seulement contre la conscription mais aussi contre la participation à la guerre. Rumilly prétend que Duplessis a été conseillé par son secrétaire Georges Léveillé (I: 538) tandis que Black soutient que l'erreur de Duplessis s'explique par un «malencontreux excès de gin et de champagne. La diction était claire, la voix forte mais il n'en était pas moins complètement ivre " (I: 346).

Il y a autant de différences entre les deux auteurs lorsqu'ils traitent des relations de Duplessis avec les femmes. Rumilly n'aborde pratiquement jamais la question sauf pour nous expliquer le célibat de Duplessis. Dans l'esprit de certains admirateurs, Duplessis était marié à la politique et il n'avait d'autre maîtresse que le Québec. Il était d'une extrême pudeur avec les femmes - du moins peut-on le croire iorsqu'on iit ie passage édifiant de Rumilily qui nous raconte: "Ḿaurice Duplessis, premier ministre ou chef de l'Opposition, ne reçoit jamais une femme seule dans son bureau sans y faire entrer quelqu'un - le plus souvent Auréa Cloutier — sous quelque prétexte.» (I: 619.) Black nous présente, lui, un Duplessis un peu moins timide ou méfiant: «Sa vie amoureuse était scandaleuse d'après les normes du temps établies par le clergé. Une de ses aventures qui semblait plus romantique que les autres se termina tragiquement par la mort de sa maîtresse dans un accident d'automobile. Il y en eut d'autres et il y eut beaucoup de «one-night stands» comme on appelait, dans l'entourage de Duplessis, ces brèves rencontres qui ne duraient qu'une nuit.» (I: 359.) Duplessis cessa de consommer des boissons alcooliques en 1943 et ses relations sentimentales furent beaucoup plus paisibles dans les quinze dernières années de sa vie. Black raconte tout de même un incident particulier où Duplessis, fort occupé avec une amie, fit attendre délibérément deux évêques dans l'anti-chambre (II: 582). Puis Black mentionne que Duplessis avait quelques amies séparées ou divorcées, et qu'il entretenait avec l'une d'elles des rapports qui «demandaient une dépense d'énergie peut-être un peu plus considérable» (II: 582). Est-ce bien nécessaire de citer le nom de cette dame, comme le fait l'auteur? Black ne paraît guère conscient du tort qu'il peut faire inutilement à la réputation de certaines personnes et à quelques reprises, il ne brille ni par son tact ni par sa délicatesse. 
Quoi qu'il en soit, en se fondant sur la même documentation, les deux auteurs nous offrent chacun un portrait intime de Duplessis passablement différent, ce qui n'est quand même pas sans importance dans une biographie! Plus encore, ce que Black nous révèle aide à comprendre pourquoi Duplessis ne s'est pas illustré dans les croisades de moralité publique.

Sur de nombreux autres aspects de la personnalité de Duplessis, cependant, les deux auteurs s'accordent parfaitement. Ils nous présentent un homme très peu du peuple, «l'avocat des ouvriers », ce qui ne l'empêche pas d'être sur la liste de paye de la Shawinigan; il est un travailleur d'une extraordinaire énergie, à la fois généreux et despotique, paternaliste et autoritaire ; c'est une bête politique à l'Assemblée comme à l'extérieur, un croyant simple et sincère, un homme qui a le culte des amis, de la famille et du passé. Les auteurs ont beaucoup d'admiration pour leur personnage et en tracent un portrait bienveillant et indulgent.

Sur les débuts proprement dits de la carrière politique de Maurice Duplessis et sur son premier gouvernement (1936-1939), les récits de Rumilly et de Black concordent assez bien sur les faits mais differrent par certaines nuances ou divergences quant à l'interprétation de l'action de Duplessis.

Le héros est décrit comme un député très actif et très habile; invinciblement, il devient chef de l'Opposition puis chef du gouvernement en 1936. Dans tous les cas, Duplessis, comme il convient, a le beau rôle et Paul Gouin, en particulier, est présenté comme un pauvre rêveur. Rumilly nous raconte, en détails, comment est né lors de l'élection de 1936 Le Catéchisme des électeurs (I: 253-); Black répète le même récit (I: 225). Les deux ignorent que Le Catéchisme des électeurs a d'abord été publié en 1935 et remanié en 1936. Rumilly soutient qu'en 1935, les conservateurs, en faisant élire 16 députés, ont mieux fait, toutes proportions gardées, que l'A.L.N. qui, à sa première tentative, en a fait élire 26 . Black suit Rumilly, et chez les deux, même en tenant compte de l'expression «toutes proportions gardées", l'interprétation est biaisée et partisane. En d'autres circonstances, Black répète Rumilly en le déformant quelque peu. Par exemple, Black écrit: "Depuis les élections, on s'inquiétait chez les libéraux. Ernest Lapointe était convaincu que la seule façon de sauver le gouvernement était de remplacer Taschereau. On tenta donc de le reléguer à la Cour Suprême où ses ancêtres l'avaient précédé, mais Taschereau refusa cette offre.» (I: 183.) Lorsqu'on parle d'offre 
refusée, on suggère très clairement qu'il s'agit d'une proposition ferme et on imagine que l'auteur s'appuie sur un témoignage ou un document pour justifier son assertion. Or, Black se contente de nous renvoyer à Rumilly (I: 210) qui avait écrit: «Des libéraux pensent à substituer Godbout ou Perrault, au premier ministre devenu trop impopulaire, et qu'on aiguillerait vers la Cour suprême.» Rumilly, quant à lui, ne réfère à aucun document, comme c'est trop souvent le cas.

Rumilly nous présente un Duplessis déjà autonomiste avant 1936; à cette image, il faut ajouter quelques nuances, même si l'auteur ne le fait pas toujours. Il comprend très bien cependant que Duplessis, en obligeant Taschereau à accepter les pensions de vieillesse, contraint ce dernier à faire un grave accroc à l'autonomie de la province et qu'un jour, cela pourrait se retourner contre lui. Black ne paraît guère comprendre ce genre de subtilités. De même, Black n'apprécie guère ceux qu'on appelait les nationalistes qui, à ses yeux ne sont que des fanatiques; Rumilly, tout en donnant tort aux nationalistes chaque fois qu'ils different d'opinion avec Duplessis, leur reconnaît quand même des qualités...

Au sujet du premier gouvernement Duplessis, Rumilly se montre généralement moins critique que Black. Le premier, contrairement au second, accorde de l'importance à ce qu'on pourrait appeler les législations symboliques de Duplessis comme la loi sur la priorité du français (qui sera retirée quelques mois après avoir été votée) ou encore la loi obligeant les compagnies exploitant les ressources naturelles à s'enregistrer au Québec. De même, Rumilly attache de l'importance à la «loi du cadenas» et à la lutte contre le communisme. Il a l'art de nous présenter la «menace» communiste comme si elle présentait un danger très réel. Au total, malgré certaines réserves très discrètes, Rumilly est très satisfait de l'œuvre de Duplessis de 1936 à 1939. Il aboutit à la conclusion que Duplessis en trois ans a fait plus que Gouin et Taschereau en quinze ans (I: 536).

Black, de son côté, défend la politique ouvrière de Duplessis, sa politique agricole, sa politique de travaux publics de même que sa politique sociale. Cependant, il reconnaît que Duplessis n'a pas pris au sérieux le programme qui l'avait fait élire; il mentionne aussi son arrogance au pouvoir, le caractère désordonné de son administration et son incroyable ineptie budgétaire. Bref, après sa défaite de 1939, Duplessis, selon Black, "avait le choix de recommencer ou de se contenter de jouer dans l'histoire du Québec le rôle d'un chef poli- 
tique secondaire sans être tout à fait insignifiant - peut-être une modeste imitation de Mercier» $(\mathrm{I}: 361)$.

Aussi bien chez Rumilly que chez Black, la technique utilisée pour construire leur récit fait problème. Il est très difficile de dégager la signification véritable des lois présentées par le gouvernement Duplessis lorsqu'on suit celui-ci de session en session. Les lois sont souvent mal expliquées, on ne sait pas si elles furent appliquées (ou on le sait à la session suivante soit plusieurs pages plus loin dans le récit). De même on ne sait pas - et c'est primordial - comment elles furent appliquées. Le lecteur doit se contenter «d'apparences» et les affirmations des auteurs sur le sens de ces lois, sans être nécessairement fausses, nous apparaissent souvent douteuses ou peu convaincantes. Pour vraiment évaluer l'œuvre de Duplessis lors de ces trois années de pouvoir, il faudra en reprendre l'étude systématiquement.

Les deux versions de l'élection de 1939 se ressemblent beaucoup. Black explique mieux le discours de Duplessis lors de l'ouverture de sa campagne à Trois-Rivières et l'impossibilité pour le gouvernement d'emprunter au début de la guerre. À ce sujet, il se réfère à deux documents sans les citer ni les analyser. Il affirme aussi que les libéraux fédéraux menaçaient les industriels de leur refuser tout contrat de guerre s'ils versaient de l'argent à l'Union Nationale (I: 351). C'est un geste spectaculaire et important mais malheureusement Black ne semble pas s'appuyer sur aucun document ou témoignage pour l'établir.

Comment expliquer la défaite de Duplessis en 1939? D'après Rumilly, c'est d'abord et avant tout à cause de la conspiration d'Ottawa; cela est logique puisque, selon lui, le gouvernement Duplessis avait été extraordinaire pendant ces trois années au pouvoir. Selon Black, plus critique vis-à-vis du régime Duplessis, il est tout aussi logique de faire appel à des causes externes et internes.

Sur la période 1939-1944 les deux auteurs perdent quelque peu la trace de leur personnage. Duplessis étant très malade à cette époque, il est souvent absent de la scène politique. De plus, en 1957, il a détruit certains documents relatifs à sa carrière. C'est ainsi que, dans l'étude de cette période, les auteurs parlent beaucoup de King, de Villeneuve et assez peu de Duplessis à certains moments.

Les deux auteurs consacrent le deuxième tome de leur ouvrage respectif aux quinze dernières années de Duplessis. Cependant, Black, 
au lieu de poursuivre son étude d'une manière chronologique comme dans le tome I, choisit une approche thématique fort différente et qui rompt l'unité de son œuvre. De son côté, Rumilly poursuit son récit comme il l'a entrepris en suivant Duplessis d'année en année, de session en session. En passant en revue les faits et gestes de Duplessis, il tente de parfaire le portrait de l'homme, de dégager les idées qui l'animent, les lignes de force de son gouvernement et de sa manière de gouverner.

Duplessis nous apparait à travers Rumilly comme un homme prudent et conservateur. Pour l'auteur - et cela vaut d'être souligné - Duplessis n'est pas nationaliste, mais il a l'instinct canadienfrançais, il incarne le Canada français. Rumilly nous rappelle souvent la générosité du "Chef»; mais assez souvent cette générosité s'exerce grâce aux fonds publics et cela ne paraît pas faire sourciller le biographe.

À l'Assemblée, aussi bien qu'au Conseil des ministres, Duplessis est le «Chef». Il a son cabinet bien en mains et d'ailleurs le Conseil des ministres «tourne au conseil de famille» (II: 129). Duplessis, en bon père de famille, sait récompenser ses ministres. Ainsi nous pouvons lire le récit de la scène suivante entre le Premier ministre et son secrétaire provincial: «Omer Côté est rentré en grâce. Duplessis lui demande: "Que veux-tu que je t'offre pour ton anniversaire?" - Nommez mon frère juge municipal. "Ce sera fait." (II: 203.)

Le régime Duplessis cultive avec un art consommé le patronage érigé en système. Rumilly est tantôt cynique, tantôt naï mais surtout partisan lorsqu'il traite ce système de l'Union Nationale et de son Chef. L'auteur a une page sublime sur Gérald Martineau, le très dévoué trésorier du parti qui "paie de sa poche ses frais de bureau, de voyage ou de secrétariat. Son magasin d'articles de bureau fournit l'administration provinciale, ce qui peut constituer une compensation. Mais il n'est pas - et Martineau y tient - le fournisseur unique.» (II: 152.) Toujours aussi édifiant, Gérald Martineau, nommé conseiller législatif, "distribue son indemnité aux petits vendeurs de journaux de Québec» (II: 432). Quant aux pratiques douteuses entourant la caisse électorale qu'administrait ce personnage, l'argument ultime de Rumilly c'est que les libéraux avaient fait la même chose au pouvoir (II: 95 et 564). D'ailleurs, il utilise le même argument pour justifier les contrats accordés sans soumission publique (II: 424) et pour excuser la pratique odieuse qui consistait à charger le Premier 
ministre ou un de ses députés de remettre personnellement aux intéressés les chèques de subvention (II: 591). À propos du refus de Duplessis d'accepter le principe d'octrois statutaires aux universités, Rumilly nous sert l'argument que le Premier ministre empêchait ainsi les universités d'accepter l'argent offert par le gouvernement fédéral et de cette façon défendait l'autonomie de la province (II: 476). Un dernier exemple, parmi bien d'autres, a trait à la scandaleuse politique d'achats de l'Union Nationale dévoilée par la Commission Salvas dans les années soixante. Rumilly reconnaît que des commissions étaient versées à des intermédiaires qui n'avaient aucunement participé aux transactions entre le gouvernement et les diverses compagnies concernées, mais il cherche à nous émouvoir en ajoutant: "Les bénéficiaires, le plus souvent de pauvres diables, ne sont pas toujours de ses partisans.» (II: 564.) Ce n'est pas faire du moralisme que de demander à l'historien qui doit décrire ce système de le faire sans chercher constamment à justifier... l'injustifiable.

Rumilly, au fil des pages, revient très souvent sur la question de l'autonomie provinciale. Toujours Duplessis a raison. L'auteur refuse de voir le côté négatif et brouillon de Duplessis. Ainsi, l'auteur soutient que si Duplessis ne rend pas public le Rapport Tremblay au moment où celui-ci lui est soumis, c'est pour ne pas nuire aux négociations fédérales-provinciales en cours à l'époque (II: 556). Que la défense de l'autonomie ait privé le Québec de certains revenus, cela semble bien établi. Cependant, cette situation ne peut justifier entièrement le conservatisme de Duplessis et son refus d'effectuer certaines réformes sous prétexte qu'à cause du fédéral, son gouvernement manquait d'argent, car au même moment, Duplessis se faisait gloire de réduire la dette de la province d'année en année. C'est une des facettes de ce que ses adversaires appelaient l'autonomie négative de Duplessis et il faut tenir compte de cet aspect lorsqu'on dresse le bilan de ses années de pouvoir.

À part la défense de l'autonomie provinciale, le régime Duplessis s'est illustré par sa politique visant au développement industriel et à la construction d'équipement collectif. Sur les «réalisations»de l'Union Nationale, Rumilly est intarissable: «Les bénédictions et inaugurations de ponts, de routes, d'écoles, d'hôpitaux se succèdent au rythme d'une, au rythme de plusieurs par semaine.» (II : 390.)

Pour nous convaincre, l'auteur ne manque pas de nous citer des chiffres qu'il veut éloquents. Mais en l'absence de toute comparaison ou d'évolution rigoureuse, que représentent les 550 ponts (II: 336) 
ou les 1105 écoles (II: 369) construits de 1944 à 1950? L'auteur ne nous facilite pas les choses lorsqu'il déclare que depuis 1945, l'U.N. a construit 4225 écoles (II: 669) tandis qu'à la page 705 , il dit que le gouvernement a fait construire plus de 2000 écoles. Les chiffres de Rumilly ressemblent à ceux qu'on peut trouver dans la propagande de l'U.N. et n'ont guère de signification, du moins à l'état brut; contrairement à ce que pense l'auteur, les faits ne parlent pas d'euxmêmes.

Cela est si vrai que Rumilly accompagne ces chiffres de jugements qui ne sont aucunement fondés mais qui semblent étayer sa thèse. Ainsi il cite Omer Côté, et l'approuve tacitement lorsque ce dernier déclare que le Québec est mieux équipé que la Californie au point de vue scolaire (II: 369). De même pour les hôpitaux: "La province de Québec, nous dit Rumilly, est la mieux organisée dans le domaine hospitalier.» (II: 659.) Plus encore, l'auteur affirme qu'à la fin de son régime, Duplessis préparait un plan d'assurance hospitalisation (II: 619), mais Rumilly ne fournit pas de preuve à l'appui de son affirmation.

Soulignons que Rumilly respecte l'axiome partisan qui veut que les ennemis de mon ami soient mes ennemis. On le constate souvent lorsqu'il traite des syndicalistes, de journalistes du Devoir ou de certains personnages comme Trudeau ou le Père Lévesque. À ce sujet, Rumilly n'est pas loin de nous présenter les Dominicains comme des agents du communisme (II: 106, 193, 195, 245). Si les Dominicains étaient des quasi-communistes, on comprend que le Québec était en grand danger et que les atteintes de Duplessis aux libertés civiles étaient pleinement justifiées!

Finalement l'ouvrage de Rumilly nous trace un portrait d'une extrême bienveillance de l'homme Duplessis. L'auteur se montre fort discret sur certains aspects de sa vie et surtout, il s'acharne à le défendre sur le plan politique. Selon Rumilly l'époque de Duplessis fut celle des «réalisations» et d'une «authentique révolution tranquille» (II : 705).

Lorsqu'en 1976, trois ans après Rumilly, Conrad Black publie une biographie de Duplessis de plus de mille pages, nous pouvions espérer qu'il irait plus loin que son devancier. Effectivement, dans le premier tome de son ouvrage, nous avons vu qu'il nous présente un Duplessis plus humain et qu'il se fait plus critique du premier 
gouvernement Duplessis. Cependant son analyse reste aussi faible que celle de Rumilly.

Dans le deuxième tome qui couvre les années 1944-1959, nous avons noté que Black modifie profondément l'approche de son récit. Dans ce tome, nous apprenons beaucoup de faits intéressants sur la vie de Duplessis, sur celle de son entourage et sur l'Union Nationale; de même que sur les relations de Duplessis avec l'épiscopat et les hommes d'affaires. À n'en pas douter, Black apporte du nouveau et sa contribution sera fort utile pour l'avenir. Mais cela ne va pas sans réserves, comme nous le verrons.

Le premier chapitre de ce deuxième tome intitulé: "Le Chef, son parti, sa cour», contient de fort intéressantes révélations sur le fonctionnement interne de l'Union Nationale. Ces éléments complétés par les notations et les documents présents dans le reste du volume nous permettent de reconstituer un tableau saisissant et infiniment plus riche de ce régime que ne le permettait le récit de Rumilly. Duplessis est le chef incontestable du parti et du gouvernement. Il a mis sur pied une machine politique extrêmement efficace qui constitue selon Black «un gouvernement parallèle» (II: 59) et qui lui permettra de rester au pouvoir pendant quinze ans. Duplessis s'informe de tout ce qui peut avoir de l'importance au point de vue électoral, et reçoit beaucoup de personnes à son bureau; il téléphone régulièrement à un grand nombre d'informateurs à travers la province et recueille des renseignements pertinents au point de vue politique; il n'exclut pas les ragots ou les médisances qui peuvent être fort utiles. Le chef a une connaissance prodigieuse de tout ce qui a trait à l'Union Nationale (II: 60). Il veille avec un soin méticuleux sur la caisse électorale (II : 13, 15, 184). Son entourage joue un rôle important de surveillance et d'information. Mais comme le Chef est très méfiant, il fait surveiller, ou surveille lui-même, ses plus proches collaborateurs, que ce soit Bégin ou Martineau (II : 47).

Duplessis domine l'Assemblée législative où «à force de manipulation il avait transformé la fonction de président en un véritable spectacle de marionnettes» (II: 570). Au comité des bills privés, c'était pire encore car il présidait lui-même: «Il trônait tel un chancelier médiéval dispensant l'équité et l'inéquité selon son bon vouloir.» (II: 575.)

Duplessis en plus de son poste de premier ministre s'est réservé le poste important de procureur général. Comme on peut le consta- 
ter à maintes reprises, Duplessis n'était guère sensible au sujet des libertés civiles des citoyens pas plus qu'il «ne se préoccupait (...) de préserver l'indépendance du pouvoir judiciaire» (II: 45). Le Premier ministre surveille tout ce qui se passe dans les divers ministères (II: 21, 567). Toutes les questions importantes sont de son ressort; le ministre des Finances est autorisé à écrire à Ottawa des lettres sans conséquence (II: 276). Duplessis dirige d'une main ferme le Cabinet. Les ministres sont punis avec une incroyable mesquinerie (II : 33, 65 note 13) ou récompensés de multiples façons (II: 29, 42) selon le bon plaisir de leur Chef.

Le Premier ministre ne pouvait pratiquement pas se résigner à démettre un de ses ministres, il préférait les surveiller étroitement, ou même effectuer leur travail avec l'aide de fonctionnaires. L'incompétence et la médiocrité navrantes de plusieurs ministres ne font guère de doute (II: $276,306,358,583$ ). De plus, nous dit Black, les ministres étaient âpres au gain (II: 61) et Duplessis savait utiliser leur ambition. On peut fortement douter que ce système était efficace, côninime le soùtientint blàck (II: 21, 62).

Dans ce même chapitre, on trouve des chiffres intéressants sur le coût des élections: 9 millions de dollars en 1956 (II: 17) et sur la caisse électorale qui, semble-t-il, contenait 18 millions de dollars à la mort du Chef (II: 13). Même s'il parait bien informé, nous aurions apprécié que l'auteur nous donne ses sources à ce sujet.

Non seulement Black nous dévoile les multiples facettes et les mécanismes du système de patronage de l'Union Nationale, mais il se porte à sa défense avec l'ardeur d'un partisan. Il soutient que le patronage est une forme de politique sociale qui, d'ailleurs, coûte moins cher aux contribuables qu'une politique sociale universelle; il note certes que le système est sélectif et permet d'acheter des votes, mais cela ne paraît pas trop grave à ses yeux. Les gens après tout étaient libres de se vendre (II: 17) et de toutes façons, Duplessis ne faisait que continuer les méthodes pratiquées par les libéraux (II: 12, 16). Le système, en plus d'être économique, était efficace. Ainsi, nous dit Black, «la fonction publique et même la police provinciale étaient très partisanes, ce qui avait l'avantage de maintenir les dépenses gouvernementales à un assez bas niveau» (II: 18). L'auteur ose même soutenir que la «parcimonie et une certaine probité furent deux constantes de l'administration Duplessis» (II: 21). Il y a dans ce chapitre un nombre effarant de demi-vérités, d'affirmations gratuites, de raisonnements tortueux et parfois même de faussetés. Un 
exemple éloquent: "La Commission Salvas instituée en 1960 pour enquêter sur les seize ans d'administration financière de l'Union Nationale n'a trouvé que deux millions de dollars dépensés mal à propos, ce qui ne constitue pas une somme exorbitante pour une si longue période, surtout si on la compare aux sommes dépensées par les régimes qui ont précédé et à ceux qui ont suivi cette période.» (II: 12.) En fait, si on examine le mandat de la Commission, on voit qu'elle était chargée d'enquêter non pas sur l'administration financière de l'U.N.; mais sur le département de la colonisation et le service des achats; l'enquête ne portait pas sur les seize ans d'administration de l'U.N., mais sur les cinq ans précédant le $1^{\text {er }}$ juillet 1960.

Après cela, on n'est guère surpris de lire sous la plume de Black que si Duplessis a duré, ce n'est pas «parce qu'il supprimait la démocratie, mais bien parce qu'il l'incarnait» (II: 17).

Dans les deux chapitres suivants, Black raconte les élections de 1948, 1952 et 1956. Il nous offre une étude partisane et impressionniste qui nous apprend peu de choses, sinon que les élections coûtaient cher, que la caisse électorale était bien garnie et que Duplessis et son parti n'hésitaient pas à recourir à la pire démagogie et à commettre d'inqualifiables entorses à la démocratie. À part l'intervention de l'U.N. dans l'élection montréalaise en 1957 que Black qualifie «d'ignoble»(II: 176), l'auteur est peu critique. Selon lui, les libéraux avaient fait la même chose au temps de Taschereau sans compter que les Canadiens français ne sont guère portés sur la démocratie.

Black a une manière tout à fait fantaisiste d'expliquer techniquement le succès de l'U.N., en divisant l'électorat québécois en cinq tranches de $20 \%$ (II: 10). Plus loin, il donne une explication un peu plus nuancée, encore que partielle et partiale: «La vraie explication [de son succès] est un mélange impérieux de patriotisme sincère, de conservatisme social, de modernisme économique et d'un penchant pour le grandiose mais qui, toutefois, n'excluait pas le terre à terre, tels la surveillance constante des polls électoraux et l'immense intérêt qu'il portait aux moindres détails concernant ses électeurs.» (II: 184.) Ailleurs l'auteur avait noté, mais en simple allusion, la prospérité de l'époque (II : 4) qui, à notre avis, doit être mise en relief pour comprendre le succès de Duplessis. Finalement, chez Black, il y a une autre explication du succès de Duplessis: c'est la psychologie du Canadien français. Ce dernier est porté au désordre (II: 15); il a besoin d'un père (II: 16). C'est ainsi que «tout un peuple remet- 
tait la direction de ses affaires temporelles à un seul homme» (II : 16). Faut-il rappeler à l'auteur qu'au sommet de sa gloire, Duplessis n'obtenait que 50 à $52 \%$ du suffrage exprimé lors des élections?

Black consacre deux chapitres à la question de l'autonomie provinciale au temps de Duplessis. L'auteur cite beaucoup de documents, nous rapporte beaucoup de faits, mais l'analyse qu'il donne des événements et de la documentation reste superficielle. Dans ce chapitre, comme dans les autres, l'esprit partisan est présent (II: 206) comme les généralisations faciles (II: 206) et les contradictions (II: 279-280). De plus, l'auteur, comme il lui arrive à l'occasion, fait fi de la chronologie lorsqu'il écrit: «Quand Saint-Laurent établit la Commission Massey, Duplessis répliqua en instituant sa propre Commission royale» (II: 267). La première Commission a été instituée en 1949 et la seconde en 1953 !

Cette section du volume se termine par une étude des relations France-Québec dont ne parle guère Rumilly. Ici encore, l'auteur nous cite des documents intéressants. Il tente de nous faire croire que Duplessis a préparé l'ouverture du Québec sur la scène internationale. Ce qui se dégage de son récit et de sa documentation est au contraire l'image d'un Duplessis très provincial. Quoi qu'il en soit, Duplessis refuse le projet qu'on lui présente d'ouvrir une maison du Québec à Paris. Mais Black écrit sans sourciller et sans donner aucune preuve: «Duplessis aurait repris le projet s'il avait vécu plus longtemps.»Et il conclut: «Après exactement deux siècles d'isolation [sic] presque continuelle, le Québec entreprenait une modeste vocation internationale.» (II: 299.) L'isolation fait chaud au cœur; les deux siècles témoignent de son ignorance; la vocation internationale, même modeste, viendra après Duplessis.

Par la suite, Black consacre deux chapitres à ce qu'il appelle les relations de l'Église et de l'État. En fait, il étudie surtout les rapports de Duplessis avec l'épiscopat. Le Père Jacques Cousineau, dans un article au Devoir (28/12/77), a réfuté certaines allégations de Black au sujet des évêques et à propos de la grève de l'amiante. Sur plusieurs points, le Père Cousineau a raison. Néanmoins, Black n'a pas eu tort d'intituler son sixième chapitre «Les évêques mangent dans ma main». Il y a assez d'éléments, dans ce chapitre et le suivant, pour nous convaincre de la dépendance de l'épiscopat vis-à-vis de Duplessis (II: 312). Que cet épiscopat ait été formé d'hommes dévoués et vertueux, nul n'en doute, mais ils se sont placés dans une situation de dépendance qui effectivement ternira le prestige et 
l'autorité de l'Église catholique du Québec; Black développe son argumentation de la manière suivante: "Durant ses années au pouvoir après 1944, Duplessis assura la prédominance de l'Église dans des domaines qui, après son règne, furent confiés au pouvoir séculier: l'éducation, les services hospitaliers et l'assistance publique (...). Ce système permettait à la province d'épargner plus d'un milliard de dollars en salaires qu'elle n'avait pas à payer. Cet argent, qui représentait quarante pour cent du budget provincial pour cette période, permettait à la province de construire écoles, hôpitaux, routes et habitations, de poursuivre l'électrification rurale, de ne pas augmenter les taxes et de maintenir sur le marché des valeurs [un bon crédit]» (II: 419). Cette dépendance est aggravée par le fait que «Duplessis dispensait le patronage aux institutions dirigées par l'Église en échange de leur docilité électorale» (II: 415). Alors que les évêques et leurs institutions paraissaient au sommet de leur gloire, ils préparaient à l'Église des lendemains difficiles.

Duplessis ne s'est vendu ni à l'Église ni aux grandes corporations. Dans le chapitre consacré au développement industriel et à la modernisation économique du Québec pendant les années 1944-1959, l'auteur veut réfuter «l'argument stupide»(II: 599) d'un Duplessis roi-nègre.

Selon Black, le mérite de Duplessis est d'autant plus grand que le régime Godbout avait laissé le Québec dans le marasme et le sousdéveloppement. L'auteur charge à fond, et il est carrément injuste en ne tenant pas compte des dix années de crise économique et de la guerre qui, tout en accroissant l'industrialisation du Québec, avait canalisé littéralement tout l'argent disponible vers l'effort de guerre. Si bien qu'au lendemain de la guerre, le Québec était affligé d'un sérieux retard dans le développement de son infrastructure économique. D'autre part, et c'est tout aussi important, le monde occidental en général et le Québec en particulier connaîtront de 1945 à 1957 une période de prospérité extraordinaire. Cela ne dépendait pas des politiques de Duplessis. L'historien impartial doit rappeler ces faits avant de dresser le bilan des années 1944-59 et d'évaluer le mérite de Duplessis et de son régime.

La pensée économique de Duplessis était très élémentaire et ce, d'autant plus qu'il se méfiait des théoriciens (II: 429). Cependant, il a élaboré un plan d'action cohérent, nous dit l'auteur. Essentiellement, il s'agissait d'attirer des capitalistes étrangers pour qu'ils investissent dans le secteur primaire, et par la suite, si possible, dans 
le secteur secondaire. À cela, s'ajoute une politique fiscale très conservatrice: budget équilibré, diminution de la dette publique, impôts et taxes au plus bas. Bien entendu, l'expansion des services sociaux et l'augmentation des salaires doivent être réduits dans la mesure du possible. Tout sera fait pour maintenir la paix sociale, en particulier, dans les relations de travail. La collaboration constante du gouvernement avec l'entreprise privée s'accompagne d'un antiétatisme et d'un anticommunisme très apprécié dans le milieu des affaires. Cette politique est en droite ligne avec celle de Gouin et de Taschereau. Duplessis n'a rien inventé en ce domaine. Black, l'homme d'affaires, est très satisfait de cette politique. Il ne lui vient pas à l'idée de mentionner que cette politique comporte des coûts.

Pour bien montrer les progrès du Québec, Black nous cite quelques pages de statistiques. Mais ce n'est pas très convaincant. Dans le tableau (p. 447), par exemple, le Québec est en-dessous de la moyenne canadienne dans les trois rubriques recensées par l'auteur; la comparaison Québec-Ontario ( $p$. 449) ne démontre pas un progrès extraordinaire du Québec; pas plus que l'évolution du réseau routier (p. 453).

Ensuite, à l'instar de Rumilly, il entonne l'hymne des «réalisations »: 2900 ponts de 1944 à 1959; 4100 écoles dans la même période; puis il y a les hôpitaux où le Québec est à l'avant-garde... Black reprend l'idée très douteuse, et qui n'est appuyée par aucune preuve, que «Duplessis songeait à établir un système d'assurance santé " (II: 455); la construction de logements va bon train (40000 dans les années cinquante) etc., etc. Avec Duplessis, le Québec connut «un progrès matériel et social incomparable et, ajoute-t-il, comme l'a écrit Robert Rumilly, ce furent Quinze années de réalisations; les faits parlent» (II: 458). Précisément, ni les faits ni les chiffres ne parlent ainsi en histoire. Il faut mettre ces statistiques en perspective, établir des points de comparaison valides, évaluer les coûts, voir si ces dépenses répondent aux besoins réels de la population, etc. Tout ce travail est à faire. Je ne prétends pas que Duplessis n'a rien fait en quinze ans; après tout il avait des dizaines, voire des centaines de millions à sa disposition et il devait être réélu à tous les quatre ans... Forcément, il a réalisé un certain nombre de choses mais pour le moment, ce que nous disent Rumilly et Black «des quinze années de réalisations»n'a d'autre valeur que celle de la propagande électorale de l'Union Nationale de l'époque. 
La deuxième partie de ce chapitre est plus modeste mais beaucoup plus intéressante et plus neuve. Il s'agit d'une analyse des relations qu'entretenait Duplessis avec certains hommes d'affaires, en particulier le multimillionnaire J. W. McConnell, propriétaire du Montreal Star.

Le Premier ministre et McConnell s'échangeaient de gros et de petits cadeaux. Peu avant les élections de 1952 et de 1956, le propriétaire du Montreal Star faisait parvenir à Duplessis personnellement 50000 à 100000 dollars, en argent comptant. Le cher homme, grand philanthrope, donnait environ 750000 dollars par année aux œuvres de charité canadiennes-françaises, choisies sous la recommandation du Premier ministre. Cependant, McConnell n'oubliait pas les siens: «Les cadeaux qu'il faisait aux projets qui lui tenaient particulièrement à cœur, notamment l'université McGill, l'hôpital Général de Montréal et l'hôpital Royal Victoria, étaient immenses et se chiffraient dans les dizaines de millions de dollars.» (II : 463.)

Duplessis, ravi par les miettes accordées à la majorité, se montrait d'une grande générosité envers les institutions anglophones et de 1946 à 1959 «les largesses du gouvernement provincial envers ces institutions coulèrent à flots comme jamais auparavant ou depuis » (II : 463).

Parlant de Duplessis, Black nous raconte que, généralement, chez les anglophones du milieu des affaires «on ricanait (...) de sa méthode excentrique et personnalisée de gouverner, on prenait un ton de délicatesse offensée pour décrire ses attitudes autoritaires, son goût de la chicane politique et son nationalisme" (II: 468). Mais l'auteur ajoute que les industriels anglophones faisaient passer leur intérêt avant leurs principes britanniques et qu'ils participaient, avec enthousiasme, au «système politico-économique byzantin de Duplessis » (II : 484).

Les anglophones pouvaient toujours compter sur Duplessis: «Ainsi la collectivité anglophone avait l'impression qu'elle n'avait pas à se préoccuper du quatre-vingt pour cent de majorité française qui les [sic] entourait. Duplessis, comme toujours, règlerait tout.» (II: 468). Et c'est Black qui qualifie de stupide l'idée d'un Duplessis roinègre, émise par Laurendeau!

On ne s'ennuie pas à lire Black, car en plus de citer abondamment des documents passionnants (qui avaient probablement échappé à Rumilly), le lecteur a droit, en prime, aux pensées profondes de 
l'auteur: "En plus d'être extraordinairement généreux, M. McConnell était très perspicace, comme l'atteste d'ailleurs la fortune considérable qu'il amassa).» (II : 467.) Le lecteur a droit aussi aux spéculations fulgurantes de l'auteur sur ce que Duplessis ferait ou ne ferait pas, s'il était au pouvoir aujourd'hui : «Il [Duplessis] n'aurait jamais toléré les politiques de ses successeurs - faire de la langue une question politique tout en exigeant du fédéral plus d'argent à dépenser au Québec avec une prodigalité partisane.» (II: 469.)

L'avant-dernier chapitre traite des rapports de Duplessis avec les ouvriers et les journalistes.

L'auteur fait état de la législation du travail votée par le gouvernement Duplessis. Le bilan est assez maigre et l'esprit qui s'en dégage, très conservateur et comme le dit Black: «Au niveau collectif, les sympathies de Duplessis allaient le plus souvent au patronat.» (II: 501.) Cela vaut, de toute évidence, non seulement pour Duplessis, mais aussi pour son biographe.

Duplessis exerçait une grande influence sur les journalistes et les propriétaires de journaux: "La presse anglaise au Québec était liée pieds et mains à Duplessis. Les journaux français, sauf Le Devoir, affichaient la même partisannerie que la presse anglaise.» (II: 520.) Black nous rapporte que l'Union Nationale a même songé à acheter Le Devoir, mais que le directeur Georges Pelletier refusa. Finalement, ajoute-t-il, c'est l'archevêché de Montréal qui s'en porta acquéreur (II: 527 et 533). Mais comme il arrive souvent chez Black, on ne sait d'où vient cette histoire puisqu'il ne mentionne aucune source. Heureusement, Le Devoir garde sa liberté. Le Premier ministre, comme son biographe, lui voue une haine féroce. Black qualifie à plusieurs reprises Le Devoir d'hystérique et cherche par tous les moyens, même les plus ignobles, à discréditer les responsables et les collaborateurs de ce journal. Ainsi, A. Laurendeau avait «un caractère instable» (II: 534); Trudeau et Pelletier sont qualifiés de «quasi intellectuels» (II: 536); Jacques Perrault était «accablé de dettes, de poursuites, mêlé à toutes sortes de tricheries financières et sentimentales, il se suicida» (II: 536); P. Laporte est pris à partie à propos d'une histoire de famille (II : 540); Black prend soin de nous rappeler qu'au moment où il termine son livre, Gérard Filion «attend son procès pour plusieurs accusations criminelles» (II: 170). On peut difficilement descendre plus bas. Et pour bien enfoncer le clou, il ajoute: «Le Devoir n'avait pas grande influence sur les électeurs de l'époque mais eut un effet considérable 
sur les historiens improvisés et malveillants.» (II : 541.) Il est assez curieux de constater que non seulement Black prend douze pages pour tenter de démolir Le Devoir mais qu'il s'en sert régulièrement comme source de documentation.

Dans le chapitre, «L'homme et la légende», Black ajoute les dernières touches au portrait du héros et fait le bilan de son œuvre. Le portrait physique de Duplessis est complet, nous avons même droit à un témoignage médical sur son pénis (II: 586). Quant à l'homme politique, son despotisme et son autoritarisme sont illustrés encore de multiples façons, mais en bon partisan, Black nous explique suavement: "Ce que la plupart de ses critiques ont appelé dictature et corruption n'étaient en réalité que les manifestations malicieuses de son amour de la plaisanterie.» (II: 579). Black en profite aussi pour lancer du vitriol à quelques «ennemis» qu'il n'avait pu attaquer précédemment. Ainsi René Lévesque nous est présenté comme un membre de la famille des politiciens ayant «le ton aigu, poussant les hauts cris à propos de tout et de rien, sermonneurs et inflexibles, [qui] parlaient le plus souvent pour énoncer des platitudes» (II : 601).

Quant au bilan de l'œuvre de Duplessis nous connaissons la thèse de l'auteur, Duplessis ne fut pas un roi-nègre, son régime ne fut pas celui de la «grande noirceur». Duplessis a contribué à la défense de l'autonomie du Québec, il a même fait du gouvernement du Québec «un gouvernement national mais non souverain» (II: 596). Avec Duplessis, «l'État du Québec devint infiniment plus important qu'il ne l'avait jamais été dans ses rapports avec l'Église, le gouvernement fédéral et l'industrie » (II: 596).

Duplessis a su réaliser une alliance entre les conservateurs et les nationalistes et mettre sur pied une machine politique d'une grande efficacité. Il a effectué une «reconstruction économique et sociale du Québec qui libéra la province des limitations d'une industrie marginale et d'une éducation insuffisante» (II: 594). Même si Duplessis était un catholique très sincère, une des conséquences de son régime fut l'effondrement de la puissance de l'Église catholique après sa mort. En s'accrochant à son rôle traditionnel dans l'éducation et les services sociaux, l'Église est devenue un organisme de distribution, voire même une succursale de l'Union Nationale; elle y a perdu son prestige et son autorité. Duplessis et l'Union Nationale, selon Black, ont trouvé là le moyen d'accomplir beaucoup à peu de frais et «la population étant généralement paisible, le gou- 
vernement put dévouer [sic] la plus grande partie de son budget bien équilibré aux travaux publics. Ceci catapulta le Québec en plein vingtième siècle en matière d'écoles, d'universités, de chemins et d'hôpitaux. Cet état de choses attira aussi le capital en un volume tel que le Québec se vit élevé du jour au lendemain au rang des économies industrielles les plus modernes du monde de l'aprèsguerre.» (II: 596.)

Certains aspects de cette interprétation du régime Duplessis ne sont pas indéfendables, mais il faudrait en faire la démonstration, avec rigueur, sans esprit partisan et sans généralisations abusives. Conrad Black ne l'a pas fait. Il ne suffit pas d'avoir accès aux archives de Duplessis et d'interviewer ceux qui l'ont connu pour écrire sa biographie et encore moins pour faire l'histoire de son époque.

Reconnaissons toutefois que, grâce à Black, nous avons appris beaucoup sur l'homme qu'était Duplessis, sur certains évêques, sur certains industriels anglo-canadiens et sur la machine de l'Union Nationale. L'information que l'on retrouve dans Black est beaucoup pius riche que celie fournie par Rumiliy qui, à maintes reprises, a fait preuve d'une trop grande discrétion... C'est dommage, car l'œuvre de Rumilly, malgré les lacunes que nous avons signalées, n'est pas sans mérite. L'auteur, sans prétention et sans arrogance, a tracé avec beaucoup d'art et de finesse un portrait discutable mais cohérent de Duplessis. Son cuvre possède une grande unité et son remarquable talent d'écrivain en rend la lecture agréable. On ne saurait en dire autant de l'ouvrage de Black qui semble taillé à la hache et qui en plus est desservi par une médiocre traduction. On pourrait relever des dizaines de fautes énormes du genre: "se montra plus conciliatoire», "l'avarice juridictionnelle», "requêtes plaintives», «région destituée», «dénigrations», «Brezil», «les industries qui demandent un travail intense», "pour labour intensive», etc...

Finalement, grâce à Black, nous pouvons espérer que désormais les archives de Duplessis seront accessibles à tous les chercheurs. Après la publication de cet ouvrage, on ne voit vraiment pas pourquoi la Société des Amis de M. Duplessis ne déposerait pas aux Archives Nationales du Québec l'ensemble de la précieuse documentation dont elle est dépositaire. Il reste beaucoup de travail à effectuer pour comprendre Duplessis et son époque. 